\title{
ARTICLE OPEN Sintilimab for relapsed/refractory extranodal NK/T cell lymphoma: a multicenter, single-arm, phase 2 trial (ORIENT-4)
}

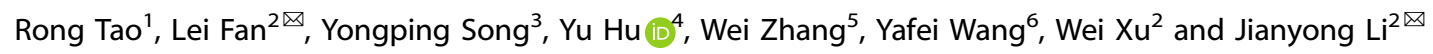

This study (ORIENT-4) aimed to assess the efficacy and safety of sintilimab, a humanized anti-PD-1 antibody, in patients with relapsed/refractory extranodal NK/T cell lymphoma ( $r / r$ ENKTL). ORIENT-4 is a multicenter, single-arm, phase 2 clinical trial (NCT03228836). Patients with r/r ENKTL who failed to at least one asparaginase-based regimen were enrolled to receive sintilimab $200 \mathrm{mg}$ intravenously every 3 weeks for up to 24 months. The primary endpoint was the objective response rate (ORR) based on Lugano 2014 criteria. Twenty-eight patients with r/r ENKTL were enrolled from August 31, 2017 to February 7, 2018. Twenty-one patients $(75.0 \%, 95 \% \mathrm{Cl}: 55.1-89.3 \%)$ achieved an objective response. With a median follow-up of 30.4 months, the median overall survival (OS) was not reached. The 24-month OS rate was $78.6 \%$ (95\% Cl, 58.4-89.8\%). Most treatment-related adverse events (TRAEs) were grade 1-2 (71.4\%), and the most common TRAE was decreased lymphocyte count (42.9\%). Serious adverse events (SAEs) occurred in 7 (25.0\%) patients, and no patient died of adverse events. Sintilimab is effective and well tolerated in patients with $r / r$ ENKTL and could be a novel therapeutic approach for the control of ENKTL in patients.

\section{INTRODUCTION}

Extranodal natural killer (NK)/T cell lymphoma (ENKTL) is a highly aggressive subtype of non-Hodgkin lymphoma (NHL) with a high incidence in Asia and Latin America. ${ }^{1}$ In China, ENKTL accounts for $6.6 \%$ of all $\mathrm{NHL}$ and $28.1 \%$ of peripheral T lymphomas (PTCLs). ${ }^{2}$ The 5-year overall survival (OS) of patients with ENKTL is $40-50 \%{ }^{1}$

ENKTL shows a poor response to conventional anthracyclinebased chemotherapy due to the high expression of the multidrug resistance P-glycoprotein. Asparaginase-based regimens are effective for ENKTL with an objective response rate (ORR) of $67-79 \%$ and a complete response (CR) rate of $45-61 \% .^{3-5}$ Patients who failed asparaginase-based therapy have limited treatment options with a median survival of $<6$ months. ${ }^{6}$

Epstein-Barr virus (EBV) infection is an important etiological and prognostic factor for ENKTL. ${ }^{7}$ Overexpression of PD-L1 induced by EBV infection is a potential mechanism for ENKTL to avert immune surveillance, and anti-PD-1 antibodies in patients with relapsed/ refractory $(r / r)$ ENKTL have shown potential efficacy. ${ }^{8}$ Anti-PD-1 monoclonal antibody $(\mathrm{mAb})$ can rescue $T$ cell viability inhibited by EBV-positive diffuse large B cell lymphoma., ${ }^{9,10}$ Two retrospective studies with limited sample size showed that pembrolizumab was active in $r / r$ ENKTL with ORRs of $100 \%(n=7)^{11}$ and $57.1 \%(n=7){ }^{12}$ respectively. Two case reports also demonstrated the efficacy of anti-PD-1 mAb in r/r ENKTL after asparaginase-based therapy. ${ }^{13,14}$ Recently, there have been a study reporting the efficacy and safety of PD-L1 antibody-avelumab monotherapy for $r / r$ NKTCL-that showed an ORR of $38 \%$ in 21 patients, with an CR rate of $24 \%{ }^{15}$
However, there is scarce clinical evidence for the antitumor activity of PD-1 inhibitors in patients with ENKTL.

Sintilimab is a recombinant humanized anti-PD-1 mAb that binds to human PD-1. Compared with pembrolizumab or nivolumab, sintilimab has a different binding site and potentially greater affinity against PD-1 according to preclinical data. ${ }^{16}$ Sintilimab has demonstrated clinical benefit in various cancers. ${ }^{17,18}$

The present prospective phase 2 study was conducted to evaluate the efficacy and safety of sintilimab against $r / r$ ENKTL.

\section{RESULTS}

Baseline characteristics

From August 31, 2017, to February 7, 2018, 34 patients were screened and 28 patients were eventually enrolled. Patients were excluded because the lesion was $<15 \mathrm{~mm}$ or not visible by ${ }^{18} \mathrm{~F}$-fluorodeoxyglucose positron emission tomography $\left({ }^{18} \mathrm{FDG}-\right.$ PET) $(n=3)$, insufficient organ or bone marrow function $(n=1)$, uncontrolled concurrent disease $(n=1)$, and active hepatitis $\mathrm{B}$ $(n=1)$ (Supplementary Fig. S1).

The median age of the patients was 37 years (range: 19-65), and $17(60.7 \%)$ were male. Nineteen patients $(67.9 \%)$ were stage IV, 25 (89.3\%) patients had an Eastern Cooperative Oncology Group (ECOG) performance status (PS) of 1 and 2. Patients received a median of $3(\mathrm{Q} 1-\mathrm{Q} 3,2.0-4.5)$ prior lines of chemotherapy, and all were relapsed or refractory to previous asparaginase-based

\footnotetext{
${ }^{1}$ Department of Hematology, Xinhua Hospital, Shanghai Jiao Tong University School of Medicine, Shanghai, China; ${ }^{2}$ The First Affiliated Hospital of Nanjing Medical University,

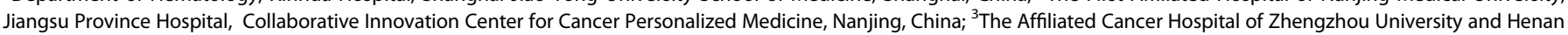

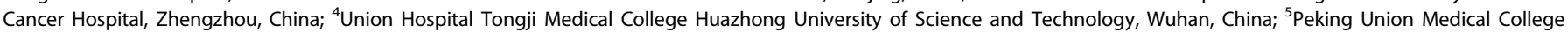
Hospital, Beijing, China and ${ }^{6}$ Tianjin Medical University Cancer Institute and Hospital, Tianjin, China

Correspondence: Lei Fan (fanlei3014@126.com) or Jianyong Li (jianyong.lijsh@outlook.com)
}

Received: 2 April 2021 Revised: 30 August 2021 Accepted: 31 August 2021

Published online: 27 October 2021 
treatment. Twenty-two (78.6\%) patients previously received radiotherapy, and 2 (7.1\%) underwent autologous stem cell transplantation (Table 1). ${ }^{19}$

\section{Response}

By February 28, 2020, the median follow-up was 30.4 months (range: 27.5-31.9). Median duration of treatment was 24.2 months (range: 1.4-33.3) and a median number of received doses was 35 (range: 2-48). Five patients who were initially determined as progressive disease (PD) finally showed a response (they were considered as pseudo-progression), and the ORR $(n=21)$ was in fact $75.0 \%$ (95\% confidence interval (Cl): 55.1-89.3) when including the 5 patients who experienced pseudo-progression prior to response. The $C R$ and partial response (PR) rates were 21.4 and $53.6 \%$, respectively. The disease control rate (DCR) was $85.7 \%$ (95\% Cl: $67.3-96.0 \%)$, including 5 patients who experienced pseudo-progression prior to response (Table 2). The first response evaluation (at 6 weeks) after enrollment of 26 patients showed 14 responders (CR $n=1$, and PR $n=13$ ), 4 patients having stable disease (SD), and several patients with early PD ( $n$ $=8$ ). Enrollment was paused due to these PD cases, but treatment was continued. Table 2 presents the responses during the whole trial. Since the lower limit of $95 \% \mathrm{Cl}$ of ORR was above the pre-specified $30 \%$ threshold, the $\mathrm{H} 1$ hypothesis for the efficacy of sintilimab was verified, and the enrollment for the present trial was terminated.

The median time to response (TTR) was 1.3 months $(95 \% \mathrm{Cl}$ : 1.3-3.4), and the median duration of response (DOR) was 4.1 months (95\% Cl: 2.1-15.2) (Fig. 1a). Subgroup analyses suggested that normal lactate dehydrogenase (LDH, $n=18$, ORR $=94.4 \%, 95 \% \mathrm{Cl}: 65.3-98.6 \%)$ and absence of bone marrow involvement $(n=22, \mathrm{ORR}=90.9 \%, 95 \% \mathrm{Cl}: 59.7-94.8 \%)$ were associated with more favorable ORR (Fig. 1b).

\section{Survival}

Till data cut-off, seven patients had death events. The 1-year OS rate was $82.1 \%$ (95\% Cl: $62.3-92.1 \%)$ and 2-year OS rate was $78.6 \%$ (95\% Cl: 58.4-89.8\%) (Fig. 2a). Five (17.9\%) patients had pseudoprogression. In these patients, the 1- and 2-year OS rates were both $100.0 \%$ (95\% Cl: 100.0-100.0), and the median OS was not reached (Fig. 2b). Fourteen (50.0\%) patients, including 5 who had pseudo-progression, showed a pattern that despite new lesions or increased ${ }^{18}$ FDG-PET uptake might be observed in asymptomatic patients after sintilimab administration, decreased uptake could be observed subsequently (Fig. 3). Among these patients, the OS rates at 1- and 2-year were both 92.9\% (95\% Cl: 59.1-99.0; Fig. 2c).

Historical controls

In the retrospective analysis, 46 patients were identified with $r / r$ ENKTL failing to asparaginase-based regimens. In these patients, ORR was $32.8 \%$, and the median OS was 4.8 months (Supplementary Fig. S2).

\section{Safety}

Twenty-seven (96.4\%) patients experienced at least one treatment-related adverse event (TRAE), with the most common TRAEs being decreased lymphocyte count (46.5\%), pyrexia (42.9\%), and decreased white blood cell count (35.7\%). Most TRAEs were grade 1-2, and only one patient experienced grade 4 TRAE (diabetes). No grade 5 TRAE was reported (Table 3). Seven (25.0\%) patients reported serious AEs (SAEs), including grade 3 pulmonary infection, anaphylactic shock, acute pancreatitis, gastrointestinal hemorrhage, intervertebral disc disorder, localized infection, ketoacidosis, and grade 2 pyrexia. Only ketoacidosis was related to sintilimab by the investigator. Two patients reported TRAE (diabetes and platelet count decreased) led to treatment discontinuation. No positive anti-drug antibody (ADA) or infusionrelated $A E$ was reported.
Table 1. Baseline characteristics of the patients

\begin{tabular}{|c|c|}
\hline Characteristics & Total $(n=28)$ \\
\hline \multicolumn{2}{|l|}{ Age, years } \\
\hline Mean \pm standard deviation & $39.8 \pm 12.67$ \\
\hline Median (range) & $37(19-65)$ \\
\hline \multicolumn{2}{|l|}{ Sex, $n(\%)$} \\
\hline Male & $17(60.7)$ \\
\hline Female & $11(39.3)$ \\
\hline \multicolumn{2}{|l|}{ Ethnic group, $n(\%)$} \\
\hline Han & $28(100.0)$ \\
\hline \multicolumn{2}{|c|}{ Time from first diagnosis, months } \\
\hline Median (Q1-Q3) & $22.0(10.0-40.2)$ \\
\hline \multicolumn{2}{|l|}{ ECOG PS, $n(\%)$} \\
\hline 0 & $3(10.7)$ \\
\hline 1 & $24(85.7)$ \\
\hline 2 & $1(3.6)$ \\
\hline \multicolumn{2}{|l|}{ Previous lines of chemotherapy } \\
\hline Median (Q1-Q3) & $3.0(2.0-4.5)$ \\
\hline$\geq 3, n(\%)$ & $15(53.6)$ \\
\hline \multicolumn{2}{|l|}{ Previous radiotherapy, $n$ (\%) } \\
\hline Yes & $22(78.6)$ \\
\hline No & $6(21.4)$ \\
\hline \multicolumn{2}{|c|}{ Previous autologous stem cell transplantation, $n(\%)$} \\
\hline None & $26(92.9)$ \\
\hline Once & $2(7.1)$ \\
\hline \multicolumn{2}{|c|}{ Asparaginase-based treatment outcome } \\
\hline Refractory & $12(42.9)$ \\
\hline Relapsed & $16(57.1)$ \\
\hline \multicolumn{2}{|c|}{ Bone marrow involvement, $n(\%)$} \\
\hline No & $22(78.6)$ \\
\hline Yes & $6(21.4)$ \\
\hline \multicolumn{2}{|l|}{ B symptoms, $n$ (\%) } \\
\hline Presence & $24(85.7)$ \\
\hline Absence & $4(14.3)$ \\
\hline \multicolumn{2}{|l|}{ Lactate dehydrogenase, $n$ (\%) } \\
\hline Normal & $10(35.7)$ \\
\hline Increased & $18(64.3)$ \\
\hline \multicolumn{2}{|l|}{ Plasma EBV, $n(\%)$} \\
\hline Positive & $8(28.6)$ \\
\hline Negative & $20(71.4)$ \\
\hline \multicolumn{2}{|l|}{ Ann Arbor stage, $n(\%)$} \\
\hline 1 & $2(7.1)$ \\
\hline II & $7(25.0)$ \\
\hline IIII & 0 \\
\hline IV & $19(67.9)$ \\
\hline \multicolumn{2}{|l|}{ PINK, $n$ (\%) } \\
\hline Low & $9(32.1)$ \\
\hline Intermediate & $11(39.3)$ \\
\hline High & $8(28.6)$ \\
\hline \multicolumn{2}{|l|}{ PINK-E, $n$ (\%) } \\
\hline Low & $16(57.1)$ \\
\hline Intermediate & $9(32.1)$ \\
\hline High & $3(10.7)$ \\
\hline
\end{tabular}

$A D A$ anti-drug antibody, EBV Epstein-Barr virus, ECOG PS Eastern Cooperative Oncology Group performance status, $P D$ progressive disease, PINK Prognostic Index for Natural Killer Lymphoma, risk factors include: age $>60$ years, stage III or IV, distant lymph node involvement, and non-nasal type disease; ${ }^{22}$ PINK-E Prognostic Index for Natural Killer Lymphoma with EBV DNA, risk factors include: age $>60$ years, stage III or IV, distant lymph node involvement, non-nasal type disease, and EBV DNA ${ }^{22}$ 


\begin{tabular}{|c|c|c|c|c|c|c|}
\hline CR & $6(21.4 \%)$ & $1(3.8 \%)$ & $1(4.0 \%)$ & $2(10.0 \%)$ & $3(15.0 \%)$ & $3(15.0 \%)$ \\
\hline PR & $15(53.6 \%)$ & $13(50.0 \%)$ & $7(28.0 \%)$ & $4(20.0 \%)$ & $0(0.0 \%)$ & $0(0.0 \%)$ \\
\hline Unevaluable & $1(3.6 \%)$ & $0(0.0 \%)$ & $0(0.0 \%)$ & $1(5.0 \%)$ & $0(0.0 \%)$ & $0(0.0 \%)$ \\
\hline Objective response (CR + PR) & $21(75 \%)$ & $53.8 \%$ & $32.0 \%$ & $30.0 \%$ & $15.0 \%$ & $15.0 \%$ \\
\hline Objective response rate $(95 \% \mathrm{Cl})$ & $55.1-89.3 \%$ & $33.4-73.4 \%$ & $14.9-53.5 \%$ & $11.9-54.3 \%$ & $3.2-37.9 \%$ & $3.2-37.9 \%$ \\
\hline Disease control $(C R+P R+S D)$ & $24(85.7 \%)$ & $69.2 \%$ & $64.0 \%$ & $60.0 \%$ & $100.0 \%$ & $100.0 \%$ \\
\hline Disease control rate $(95 \% \mathrm{Cl})$ & $67.3-96 \%$ & $48.2-85.7 \%$ & $42.5-82.0 \%$ & $36.1-80.9 \%$ & $83.2-100.0 \%$ & $83.2-100.0 \%$ \\
\hline
\end{tabular}

\section{Quality of life}

Quality of life of the enrolled patients was significantly improved, starting at week 15. For the EuroQol-5-Dimensional-5-level (EQ$5 \mathrm{D}-5 \mathrm{~L}$ ) index, the mean score increased from 0.8 at baseline to 0.9 at week $15(P<0.05)$ and then remained at 0.9 to Week 96 (Supplementary Fig. S3A). For EQ-5D-5L Vas, the mean score increased from 79.3 at baseline to 90.6 at week $24(P<0.0001)$ and then remained between 87.9 and 91.8 from weeks 24 to 96 (Supplementary Fig. S3B). For QLQ-C30, the mean score increased from 70.5 at baseline to 85.4 at week $24(P<0.0001)$ and then remained between 82.0 and 87.3 from weeks 24 to 96 (Supplementary Fig. S3C).

\section{DISCUSSION}

This phase 2 study showed that sintilimab was effective and well tolerated in patients with $\mathrm{r} / \mathrm{r}$ ENKTL.

Originally, we planned to enroll 20-60 patients and a minimum sample size of 43 was required based on the statistical analysis. However, multiple early PD cases were observed after enrolling 28 patients, and the evaluation on response became difficult per Lugano 2014 criteria.

Therefore, enrollment was paused and treatment was continued to confirm the true nature of the pseudo-progression according to the investigator's discretion. After 3 months of observation and in-depth analysis of 28 patients, sintilimab demonstrated a $75.0 \%$ ORR when taking pseudo-progression into account. Under the hypothesis that the 15 yet-to-be-enrolled patients would be non-responders, there would be 19 responders out of 43 overall patients (28 actually enrolled +15 fictive), providing a $95 \% \mathrm{Cl}$ of $30.4-58.9 \%$ by Wilson's method, where the lower boundary was still greater than the predefined $30 \%$ threshold. From the view of proof of concept, the efficacy of sintilimab in $r / r$ ENKTL patients was proved.

The standard treatment for ENKTL after the failure of the asparaginase-based regimen has not been established. ${ }^{20}$ The multidrug resistance-independent DeVIC regimen has been suggested for ENKTL, but this strategy is currently used to combine with radiotherapy, and there is no data regarding $r / r$ ENKTL. ${ }^{21}$ Gemcitabine-containing regimen for $r / r$ ENKTL failing to L-asparaginase-based therapy achieved an ORR of $40 \%$ but had a dismal long-term outcome. ${ }^{22}$ No data are available about the application of other drugs such as histone deacetylase inhibitors and anti-CD30 mAbs for ENKTL, which are effective in PTCL.

In the ORIENT-4 study, the ORR for sintilimab in $r / r$ ENKTL was 75.0\% (95\% Cl: $55.1-89.3 \%)$, and the median DOR was 4.1 months (range: $2.1-15.2)$. In previous small-scale studies $(n=7)$, the ORRs of anti-PD-1 antibody in $\mathrm{r} / \mathrm{r}$ ENKTL ranged from 57.1 to $100 \% .^{12,13}$ The retrospective analysis based on the clinical data in 46 patients with $\mathrm{r} / \mathrm{r}$ ENKTL here showed a lower ORR of $32.8 \%$. A recently published phase 2 study conducted by Professor Won Seog Kim's team showed that anti-PD-L1 mAb-avelumab monotherapyachieved an ORR of $38 \%$ and CR rate of $24 \%$ in $r / r$ ENKTL patients. Comparing this study to the present study, the sample was similar (21 vs 28 ), the CR rate was comparable ( 24 vs $21.4 \%$ ), but the ORR was significantly different (38 vs $75 \%$ ); one possible reason might be that sintilimab was administered after PD at the investigator's discretion, and most patients who experienced PD eventually received sintilimab. This was significantly different from the phase 2 study of avelumab, in which patients withdrew from study immediately after PD. ${ }^{15}$ No previous study reported DOR and long-term survival of anti-PD-1/PD-L1 antibody in $r / r$ ENKTL. Efficacy data of ORIENT-4 demonstrated that sintilimab was effective in patients with $r / r$ ENKTL.

In the present study, the long-term efficacy of sintilimab was favorable in $\mathrm{r} / \mathrm{r}$ ENKTL, with a 1-year OS rate of $82.1 \%$ and a 2-year OS rate of $78.6 \%$, and the median OS was not reached. Previous retrospective analysis showed that the median OS was 6.4 months in comparable patient cohort of $r / r$ ENKTL ${ }^{6}$ and $r / r$ PTCL (including ENKTL, 6.5 months). ${ }^{23}$ Therefore, these results suggested that the current salvage treatments for $r / r$ ENKTL are inadequate, and sintilimab could be one helpful treatment strategy. We suggest that the follow-up time could be 16-34 weeks for the evaluation of ORR, which lasts for about 6 months. Still, for $r / r$ ENKTL patients with no evidence of PD, a longer follow-up time could have a higher clinical significance.

During this study, 5 (17.9\%) patients were observed having pseudo-progression, and 14 (50.0\%) patients showed a fluctuant response. Of all the patients with pseudo-progression (100\%), their 1- and 2-year OS rates were relatively greater than the overall population. In addition, the median OS was not reached, demonstrating no association between pseudo-progression or fluctuant response and an inferior OS. This suggests that the phenomenon of pseudo-progressions or fluctuant response should be taken into account during sintilimab treatment and must be confirmed before treatment alteration. Failing to confirm the true nature of the apparent PD could lead to early cessation of effective treatment and decrease possible benefits of patients.

Pseudo-progression is not rare when treating patients with immune checkpoint inhibitors. In a previous study on solid tumors treated with pembrolizumab $(n=655)$, the early pseudo-progression 
a

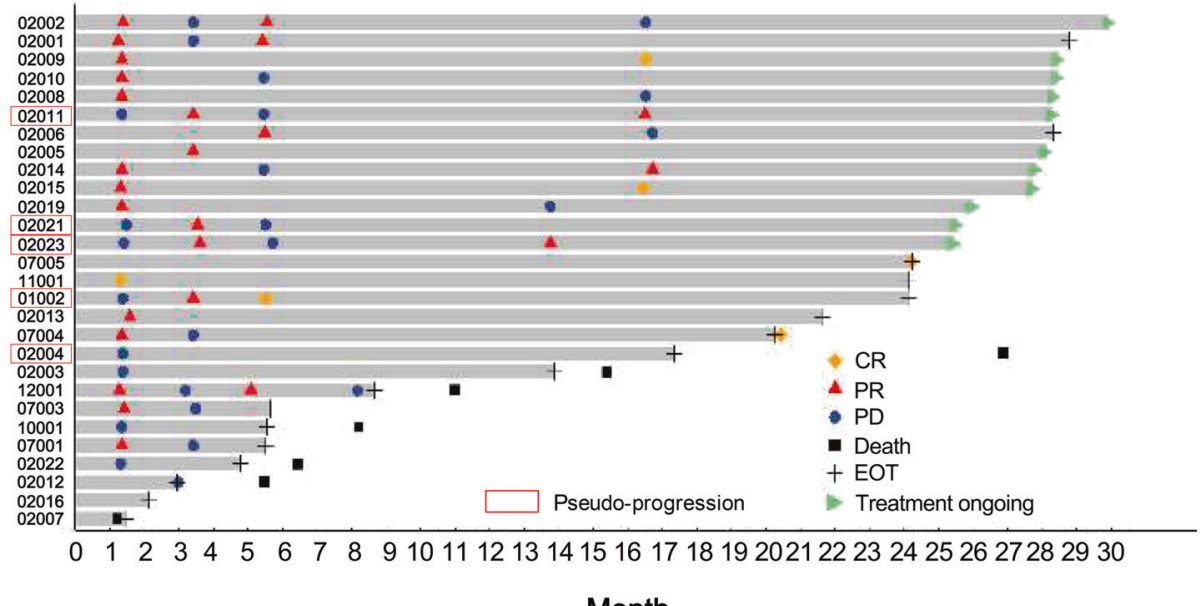

Month

b

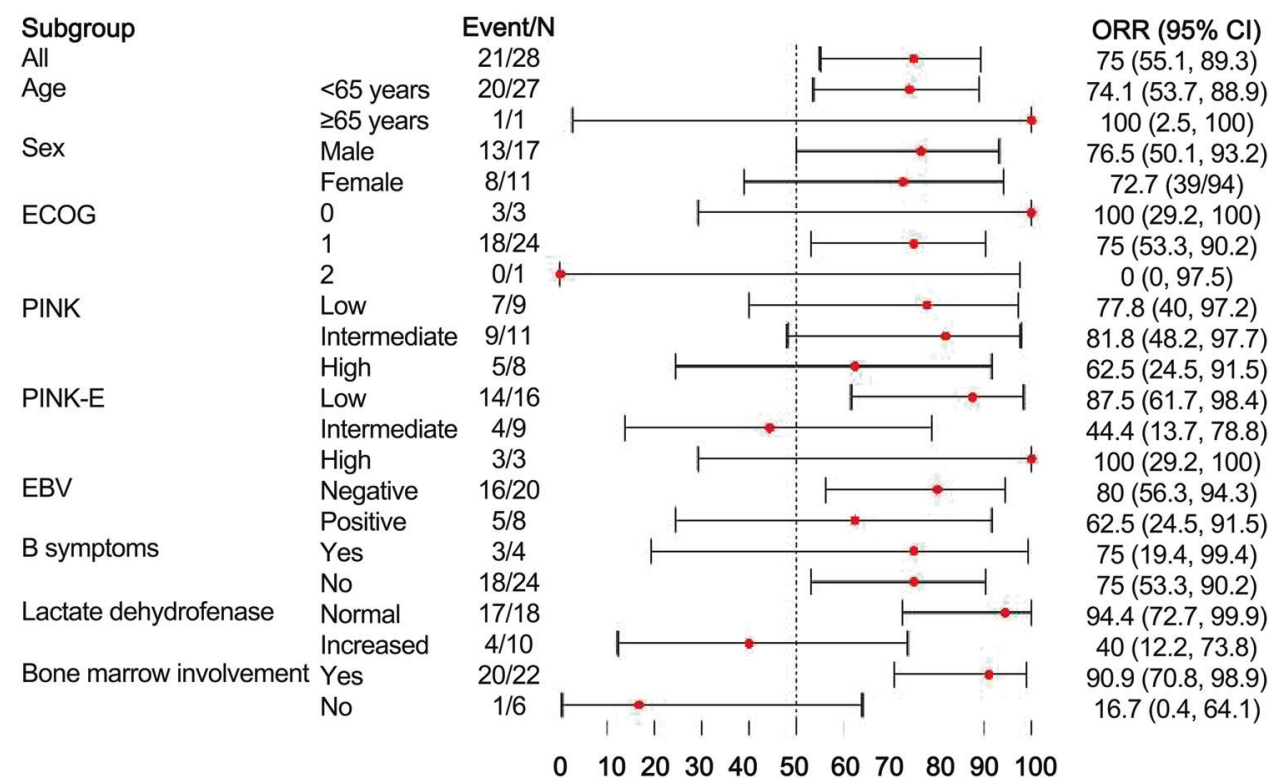

Fig. 1 Response to sintilimab in patients with relapsed/refractory NK/T cell lymphoma. a Analysis of time to response and duration of response. Patient 02004 was evaluated as stable disease after experiencing pseudo-progression. b Subgroup analysis of ORR

and delayed pseudo-progression was reported in 5 and $3 \%$ of patients, respectively. ${ }^{24}$ Kwong et al. reported one of the seven pseudo-progression cases in patients with $r / r$ ENKTL treated with pembrolizumab. ${ }^{13}$ In the present study, the response pattern was displayed as transient flares in different nodal groups without overall progression, which was consistent with the response pattern observed with nivolumab for Hodgkin lymphoma. ${ }^{25}$ For the study design in 2016, the Lugano 2014 criteria were recommended for NHL evaluation, but it lacked the criteria for pseudo-progression in lymphoma response assessment. In this study, it was observed that pseudo-progression or fluctuant response is not rare in ENKTL, thus Lugano 2014 criteria may not be the best option for this trial. Although 16-24 weeks is the recommended time point for ORR evaluation after standard treatment in patients with $\mathrm{NHL}$, long-term observation and an extended time point of 6 months and 1 year should be recommended for ORR evaluation in patients with $r / r$ ENKTL lacking obvious disease progression. In this study, several patients achieved PR or CR after $>1$ year of treatment. Nevertheless, differentiating pseudo-progression from true progression is a major challenge since there is no uniform definition of pseudo- progression. ${ }^{11,26-28}$ Additional studies are necessary to identify reliable markers, and our limited experience of identification of pseudo-progression are: (1) repeated imaging examination at longer evaluation time point; (2) serological markers like $\mathrm{LDH}$, ferritin, and sCD25; (3) circular EBV DNA copy number load; (4) clinical symptoms and quality of life scores. Regarding the time point for the evaluation of treatment efficacy, NHL has suggested that imaging evaluation should be performed at 4-6 months. Nevertheless, the findings of this study suggest that the treatment with checkpoint inhibitors in ENKTL might induce a relatively long-time pseudo-progression. The definite time point for the evaluation of treatment efficacy could not be concluded in this study. Our experience suggests that the time point of evaluation should be later than 6 months.

In the present study on sintilimab monotherapy, the DCR (85.7\%) was excellent, but the deep response rate (CR 21.4\%) was relatively low, suggesting that combination therapy might be needed to improve the CR rate. Several studies are ongoing on the use of anti-PD-1 antibodies in combination with chemotherapy (NCT04004572, NCT03701022, and NCT03936452), and we look forward to their results for further information. 
a

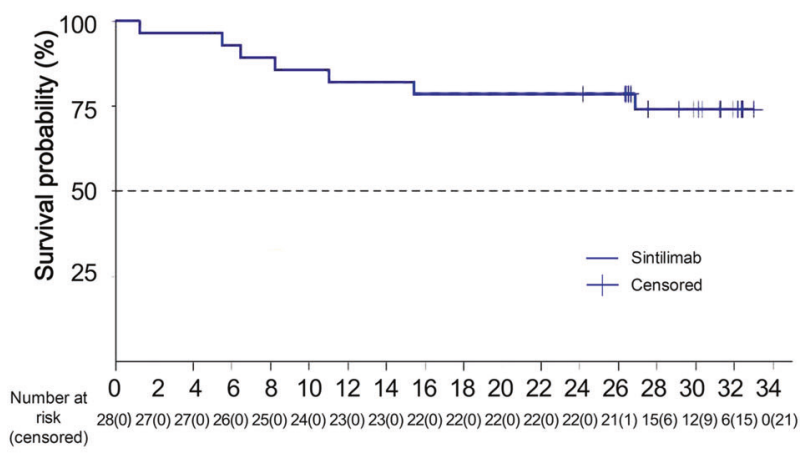

b

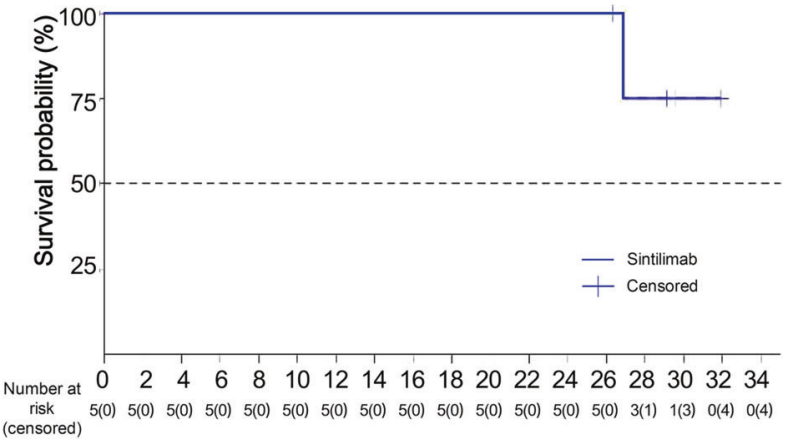

C

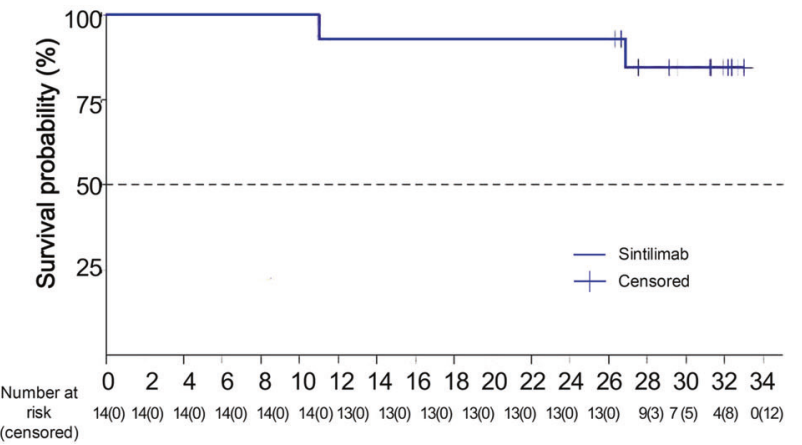

Fig. 2 Overall survival with sintilimab in patients with NK/T cell lymphoma. a Overall survival in all patients (28 patients) ( $X$-axis refers to time of follow-up in months). b Overall survival in patients with pseudo-progression ( 5 patients) ( $X$-axis refers to time of followup in months). c Overall survival in patients with the response pattern displayed as transient flares in different nodal groups without overall progression in the original target lesions (14 patients) ( $X$-axis refers to time of follow-up in months)

In the present study, all patients experienced at least one $A E$, but no grade $5 \mathrm{AE}$ was observed. The most common TRAEs were decreased lymphocyte count $(46.4 \%)$, pyrexia $(42.9 \%)$, and decreased white blood cell count (35.7\%). This was globally consistent with the results of the ORIENT-1 study, ${ }^{17}$ in which $93 \%$ of the patients had at least one $A E$, and $15 \%$ had SAEs. ${ }^{17}$ In addition, this safety profile was generally more favorable than that observed for chemotherapy in patients with ENKTL, in which grade 3-4 AEs were more common than those observed with sintilimab. ${ }^{3-5}$ Overall, the safety profile was consistent with that of other PD-1 targeted therapies in similar patients. ${ }^{11,17,18,29,30}$

The present study also has some limitations. First, it was a single-arm study without any direct control. Only historical controls from a single center were used. Second, the outcomes were assessed by investigators, which might have a bias on the evaluation. Third, the response criteria were based on Lugano 2014, which might not be the best option for patients with ENKTL treated with an immune checkpoint inhibitor. Fourth, the patients received a wide variety of treatments after this study. The treatments were too diverse for further analysis.

A major limitation is that the study did not reach its prespecified sample size. Indeed, the study was stopped temporarily due to the large number of cases with PD at 6 weeks $(n=9)$, of whom 4 cases were finally ruled out to be pseudo-progression, and the censoring time was already reached when the patients were confirmed with pseudo-progression. Therefore, only 28 patients were enrolled in this study. In addition, response evaluation at 6 weeks was probably not adequate for determining the ORR. Still, according to the actual ORR in this study, as well as $a=0.05$, power $=80 \%$, and historical control of $30 \%$, a post hoc power analysis showed that the sample size required would be 10 . If the historical control were $40 \%$, the sample size required would be 23. In this study, 28 patients were enrolled when the enrollment was stopped, which could meet the statistical requirement. As this study is sufficient to reach a conclusion, no further studies were performed.

In conclusion, sintilimab is effective and well tolerated in patients with $r / r$ ENKTL and can be a promising treatment option. Further studies are necessary to determine the exact benefit for these patients treated with sintilimab-based regimens.

\section{MATERIAL AND METHODS}

Study design and participants

This multicenter single-arm phase 2 clinical study (ORIENT-4) was conducted at six hospitals in China (NCT03228836). Patients aged between 18 and 70 years, with histopathologically confirmed ENKTL and with at least one measurable lesion $(>15 \mathrm{~mm}$ or positive ${ }^{18}$ FDG-PET uptake) were enrolled. Other major inclusion criteria were ECOG PS of $0-2$, adequate organ and bone marrow functions, had received at least one prior asparaginase-based chemotherapy (stage I/II patients must have been treated with local radiotherapy), and either relapsed or refractory to previous treatments. Relapse is defined as new lesions at the primary location or other sites after achieving CR; refractory is defined as any of the following: PD after two treatment cycles, not achieving a PR, or CR after four or six treatment cycles, respectively.

The major exclusion criteria included: aggressive NK cell leukemia, primary or secondary central nervous system lymphoma, severe hemophagocytic syndrome at initial diagnosis of ENKTL-NT, and previous exposure to any checkpoint inhibitors. The complete eligibility criteria are shown in Supplementary Materials.

Retrospective data of patients with $r / r$ ENKTL to asparaginasebased regimens were collected from the database of Jiangsu Province Hospital (Nanjing, China) as historical controls. The inclusion criteria were (1) patients with $r / r$ ENKTL; (2) failed L-asparaginase therapy.

This study was approved by the ethics committee of each participating center and was registered at www.clinicaltrials.gov (NCT03228836). It was conducted in accordance with the principles of the Declaration of Helsinki and the Good Clinical Practice guideline. All patients provided written informed consent prior to any study procedure.

\section{Procedures}

The eligible patients received sintilimab $200 \mathrm{mg}$ intravenously every 3 weeks until PD, death, intolerable toxicity, or withdrawal of informed consent, up to 24 months. Treatment beyond PD was allowed according to the investigator's discretion. 

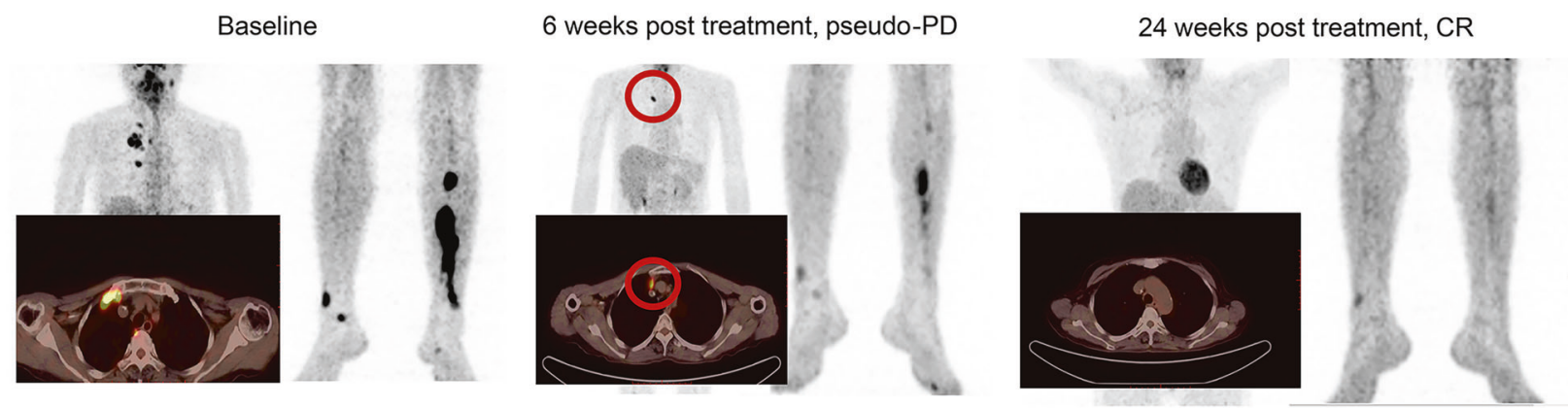

Fig. 3 Patterns of radiologic tumor pseudo-progression after sintilimab. This picture shows CT imaging of a 59-year-old female patient in stage IV involving the head and neck area, mediastinum, and shank (left). This patient was first diagnosed with ENKTL in 2017 and previously received three cycles of L-asparaginase plus gemcitabine and oxaliplatin (GELOX) followed by local radiotherapy (50 Gy). On January 31,2018 , after progressive disease, the patient participated in ORIENT-4 and received sintilimab $200 \mathrm{mg}$ Q3W. Six weeks after treatment, most lesions regressed, while a new lesion appeared on the right mediastinal pleura (middle), which had not been observed before. Twenty-four weeks after treatment, CT imaging showed further regression of right mediastinal pleura lesions, with the appearance of new lesions in week 6 ; the patients achieve a complete response (right). The areas marked by red cycles were new lesions

\begin{tabular}{|lll|}
\hline Table 3. Most common TRAEs & & \\
\hline Treatment-related adverse events, $n$ (\%) & \multicolumn{1}{l|}{ All patients $(N=28)$} \\
\cline { 2 - 3 } & Grade 1-2 & Grade 3 \\
\hline Any TRAE & $17(60.7)$ & $11(39.3)$ \\
Lymphocyte count decreased & $12(42.9)$ & $2(7.1)$ \\
White blood cell count decreased & $12(42.9)$ & 0 \\
Pyrexia & $12(42.9)$ & 0 \\
Hypothyroidism & $9(32.1)$ & 0 \\
Blood thyroid-stimulating hormone increased & $7(25.0)$ & 0 \\
Blood glucose increased & $7(25.0)$ & 0 \\
Upper respiratory tract infection & $6(21.4)$ & $1(3.6)$ \\
Hemoglobin decreased & $6(21.4)$ & 0 \\
Platelet count decreased & $5(17.9)$ & $1(3.6)$ \\
Globulin increased & $5(17.9)$ & 0 \\
Blood alkaline phosphatase increased & $5(17.9)$ & 0 \\
Nasosinusitis & $5(17.9)$ & 0 \\
Urinary tract infection & $5(17.9)$ & 0 \\
\hline Listed are any TRAEs occurring in $\geq 15 \%$ patients & & \\
TRAE treatment-related adverse event & & \\
\hline
\end{tabular}

The patients were evaluated for efficacy using ${ }^{18} \mathrm{FDG}$-PET at baseline and weeks 6, 15, and 24. Contrast-enhanced computed tomography was used at baseline and weeks 24,36 , and 48 and every 24 weeks thereafter. Efficacy was assessed according to the Lugano 2014 standard. ${ }^{31}$ The best overall response was the best response recorded from the start of the study until progression/ relapse. Safety was monitored throughout the study until 90 days after the last dose of sintilimab. The quality of life was analyzed at the initial dose, at weeks $6,15,24,36$, and 48 , and every 24 weeks thereafter. The blood samples for ADA and neutralizing antibody (NAb) were collected at baseline and just before sintilimab administration at weeks 3 and 9 and every 12 weeks thereafter. $\mathrm{NAb}$ has been reported for sintilimab. ${ }^{16}$

During the trial, for patients with PD, the investigators comprehensively judged from their radiographic results, laboratory results, and clinical performance, and some were evaluated as being in pseudo-progression. For these patients, treatment was given continuously, and investigators continue to observe and follow up these patients in accordance with the requirements of the clinical trial design.

\section{Outcomes}

The primary endpoint was the $\mathrm{ORR}(\mathrm{CR}+\mathrm{PR})$ of sintilimab monotherapy for $r / r$ ENKTL. The secondary endpoints included CR and PR rates, DCR (CR + PR + SD), TTR, DOR, 1-year OS, safety, and quality of life. Reported adverse events were graded based on the Common Terminology Criteria for Adverse Events (Version 4.03). The quality of life was evaluated using the EQ-5D-5L scale and the European Organization for Research and Treatment of Cancer Quality-of-Life Questionnaire-Core 30 (EORTC QLQ-C30).

\section{Statistical analysis}

It was planned to enroll 20-60 r/r ENKTL patients. The ORR was set to $\leq 30 \%$ in the null hypothesis using a two-sided $a$ of 0.05 . To reject the null hypothesis and achieve an expected ORR of $50 \%$ with an $80 \%$ power, a minimum of 43 patients was required. If the lower boundary of the ORR $95 \% \mathrm{Cl}$ was $>30 \%$, the efficacy of sintilimab monotherapy for $r / r$ ENKTL was demonstrated. The choice of $30 \%$ ORR was based on the historical ORR data for $r / r$ NK/T and $r / r$ PTCL. Patients with $r / r$ ENKTL reported a $40 \%$ ORR after gemcitabine-based treatment ${ }^{22}$ and achieved a 15\% ORR after chidamide monotherapy. ${ }^{32}$ Patients with $\mathrm{r} / \mathrm{r}$ PTCL obtained an ORR of $28 \%$ after asparaginase-based regimen in a Chinese population. $^{33}$

The binomial distribution was used to estimate ORR, CR, PR, and DCR and the 95\% Cls. Kaplan-Meier method was applied to estimate the time-to-event endpoints (DOR and OS) and their $95 \%$ $\mathrm{Cl}$. For all analyses, $P$ values $<0.05$ were considered statistically significant. All analyses were carried out using SAS 9.4 (SAS Institute, NY, USA).

\section{DATA AVAILABILITY}

All data or resources used in the paper are available by reasonable requirements to the corresponding authors L.F. (fanlei3014@126.com) and J.L. (jianyong.lijsh@outlook. com).

\section{ACKNOWLEDGEMENTS}

The authors thank the patients and their families, as well as the participating study teams, for making this study possible. This trial was supported by the National Natural Science Foundation of China (81720108002), the National Science and Technology Major Project (2018ZX09734-007), Excellent Youth Foundation Project of Jiangsu Province (Grant No.BK20160099), Translational Research Grant of NCRCH (2020ZKZB01), and CSCO Research Foundation (Y-Roche2019/2-0090).

\section{AUTHOR CONTRIBUTIONS}

J.L. and L.F.: study design, data collection and analysis, statistical analysis, and manuscript drafting. R.T.: study design and data collection and analysis. Y.S., Y.H., W. 
Z., Y.W., and W.X.: data collection and critical revision of the manuscript. All authors have approved the manuscript submission.

\section{ADDITIONAL INFORMATION}

Supplementary information The online version contains supplementary material available at https://doi.org/10.1038/s41392-021-00768-0.

Competing interests: The authors declare no competing interests.

\section{REFERENCES}

1. Haverkos, B. M. et al. Extranodal NK/T cell lymphoma, nasal type (ENKTL-NT): an update on epidemiology, clinical presentation, and natural history in North American and European cases. Curr. Hematol. Malig. Rep. 11, 514-527 (2016).

2. Li, X., Li, G., Gao, Z., Zhou, X. \& Zhu, X. The relative frequencies of lymphoma subtypes in China: a nationwide study of 10002 cases by the Chinese Lymphoma Study Group. Ann. Oncol. 22, iv142 (2011).

3. Yamaguchi, M. et al. Phase I study of dexamethasone, methotrexate, ifosfamide, L-asparaginase, and etoposide (SMILE) chemotherapy for advanced-stage, relapsed or refractory extranodal natural killer (NK)/T-cell lymphoma and leukemia. Cancer Sci. 99, 1016-1020 (2008).

4. Yamaguchi, M. et al. Phase II study of SMILE chemotherapy for newly diagnosed stage IV, relapsed, or refractory extranodal natural killer (NK)/T-cell lymphoma, nasal type: the NK-Cell Tumor Study Group study. J. Clin. Oncol. 29, 4410-4416 (2011).

5. Jaccard, A. et al. Efficacy of L-asparaginase with methotrexate and dexamethasone (AspaMetDex regimen) in patients with refractory or relapsing extranodal NK/T-cell lymphoma, a phase 2 study. Blood 117, 1834-1839 (2011).

6. Lim, S. H. et al. Beyond first-line non-anthracycline-based chemotherapy for extranodal NK/T-cell lymphoma: clinical outcome and current perspectives on salvage therapy for patients after first relapse and progression of disease. Ann. Oncol. 28, 2199-2205 (2017).

7. Harabuchi, Y., Takahara, M., Kishibe, K., Nagato, T. \& Kumai, T. Extranodal natural killer/T-cell lymphoma, nasal type: basic science and clinical progress. Front. Pediatr. 7, 141 (2019).

8. Jo, J. C. et al. Expression of programmed cell death 1 and programmed cell death ligand 1 in extranodal NK/T-cell lymphoma, nasal type. Ann. Hematol. 96, 25-31 (2017).

9. Quan, L. et al. PD-1 blockade can restore functions of T-cells in Epstein-Barr virus-positive diffuse large B-cell lymphoma in vitro. PLOS ONE 10, e0136476 (2015).

10. Fang, W. et al. EBV-driven LMP1 and IFN-gamma up-regulate PD-L1 in nasopharyngeal carcinoma: Implications for oncotargeted therapy. Oncotarget 5, 12189-12202 (2014).

11. Kwong, Y. L. et al. PD1 blockade with pembrolizumab is highly effective in relapsed or refractory NK/T-cell lymphoma failing L-asparaginase. Blood 129, 2437-2442 (2017).

12. Li, X. et al. Activity of pembrolizumab in relapsed/refractory NK/T-cell lymphoma. J. Hematol. Oncol. 11, 15 (2018).

13. Lai, J., Xu, P., Jiang, X., Zhou, S. \& Liu, A. Successful treatment with antiprogrammed-death-1 antibody in a relapsed natural killer/T-cell lymphoma patient with multi-line resistance: a case report. BMC Cancer 17, 507 (2017).

14. Asif, S. et al. Pembrolizumab in newly diagnosed EBV-negative extranodal natural killer/T-cell lymphoma: a case report. Mol. Clin. Oncol. 10, 397-400 (2019).

15. Kim, S. J. et al. Avelumab for the treatment of relapsed or refractory extranodal NK/T-cell lymphoma: an open-label phase 2 study. Blood 136, 2754-2763 (2020).

16. Wang, J. et al. Durable blockade of PD-1 signaling links preclinical efficacy of sintilimab to its clinical benefit. MAbs 11, 1443-1451 (2019).
17. Shi, Y. et al. Safety and activity of sintilimab in patients with relapsed or refractory classical Hodgkin lymphoma (ORIENT-1): a multicentre, single-arm, phase 2 trial. Lancet Haematol. 6, e12-e19 (2019).

18. Xu, J. M. et al. A first-in-human phase 1a trial of sintilimab (IBI308), a monoclonal antibody targeting programmed death-1 (PD-1), in Chinese patients with advanced solid tumors. J. Clin. Oncol. 35, e15125 (2018).

19. Kim, S. J. et al. A prognostic index for natural killer cell lymphoma after nonanthracycline-based treatment: a multicentre, retrospective analysis. Lancet Oncol. 17, 389-400 (2016).

20. Horwitz, S. M. et al. T-Cell Lymphomas. Version 2.2019 (National Comprehensive Cancer Network, 2018)

21. Makita, S. \& Tobinai, K. Clinical features and current optimal management of natural killer/T-cell lymphoma. Hematol. Oncol. Clin. North Am. 31, 239-253 (2017).

22. Ahn, H. K. et al. Gemcitabine alone and/or containing chemotherapy is efficient in refractory or relapsed NK/T-cell lymphoma. Invest. N. Drugs 31, 469-472 (2013).

23. Mak, V. et al. Survival of patients with peripheral T-cell lymphoma after first relapse or progression: spectrum of disease and rare long-term survivors. J. Clin. Oncol. 31, 1970-1976 (2013).

24. Hodi, F. S. et al. Evaluation of immune-related response criteria and RECIST v1.1 in patients with advanced melanoma treated with pembrolizumab. J. Clin. Oncol. 34, 1510-1517 (2016).

25. Cheson, B. D. et al. Refinement of the Lugano Classification lymphoma response criteria in the era of immunomodulatory therapy. Blood 128, 2489-2496 (2016).

26. Elnair, R. \& Lunning, M. A. Checkpoint inhibition in ENKTL: Kno_le_ge G_ps. Blood 136, 2721-2722 (2020).

27. Ma, Y., Wang, Q., Dong, Q., Zhan, L. \& Zhang, J. How to differentiate pseudoprogression from true progression in cancer patients treated with immunotherapy. Am. J. Cancer Res. 9, 1546-1553 (2019).

28. Reardon, D. A. \& Weller, M. Pseudoprogression: fact or wishful thinking in neurooncology? Lancet Oncol. 19, 1561-1563 (2018).

29. Kwong, Y. L., Lopes, D. \& Khong, P. L. Low-dose pembrolizumab induced remission in patients with refractory classical Hodgkin lymphoma. Br. J. Haematol. 176, 131-132 (2017).

30. Ansell, S. M. Sintilimab: another effective immune checkpoint inhibitor in classical Hodgkin lymphoma. Lancet Haematol. 6, e2-e3 (2019).

31. Van Heertum, R. L. et al. Lugano 2014 criteria for assessing FDG-PET/CT in lymphoma: an operational approach for clinical trials. Drug Des. Dev. Ther. 11, 1719-1728 (2017).

32. Shi, Y. et al. Results from a multicenter, open-label, pivotal phase II study of chidamide in relapsed or refractory peripheral T-cell lymphoma. Ann. Oncol. 26, 1766-1771 (2015).

33. Shi, Y. et al. Chidamide in relapsed or refractory peripheral T cell lymphoma: a multicenter real-world study in China. J. Hematol. Oncol. 10, 69 (2017).

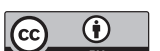

Open Access This article is licensed under a Creative Commons Attribution 4.0 International License, which permits use, sharing, adaptation, distribution and reproduction in any medium or format, as long as you give appropriate credit to the original author(s) and the source, provide a link to the Creative Commons license, and indicate if changes were made. The images or other third party material in this article are included in the article's Creative Commons license, unless indicated otherwise in a credit line to the material. If material is not included in the article's Creative Commons license and your intended use is not permitted by statutory regulation or exceeds the permitted use, you will need to obtain permission directly from the copyright holder. To view a copy of this license, visit http://creativecommons. org/licenses/by/4.0/.

(c) The Author(s) 2021 